\title{
The Medical Curriculum.
}

\section{QUALIFICATION AND REGISTRATION.}

THe first thing a medical student has to do is to register himself as such, because the five years of study which are necessary in order to obtain a qualification are dated from the time of registration. For this purpose application must be made to the Registrar of the General Medical Council, 299 Oxford Street, London, W.

The student, however, cannot be registered until he has (1) passed a preliminary examination in Arts which is recognised by the General Medical Council, and (2) has commenced medical study.

A complete list of the recognised preliminary examinations may be obtained on application to the Registrar of the General Medical Council; and it is essential in any case to discover at a sufficiently early period what preliminary examinations are recognised by the particular examining body that it is proposed to work under. For example, with few exceptions, the London Matriculation is the only preliminary examination which will permit a candidate to proceed to the higher examinations in order to become a graduate of the University of London.

Wherever he proposes to study and whatever qualification he intends to get, the courses of study which a medical student has to undergo are really very much the same; they may be divided, generally speaking, into the two groups of the preliminary and the clinical.

But it is not, as a rule, compulsory to undergo the necessary courses of instruction in these two divisions of medical education at the same school. Often a student will be well advised to undergo his preliminary training at a provincial school, or as a student of the University of London, and to pursue his clinical studies elsewhere, as in one of the large Metropolitan hospitals.

The following is a list of the examining bodies and the degrees and diplomas granted by them which admit the graduate or diplomate to enter his name in the register of legally qualified medical men:ENGLAND.
Examining Body. University of Oxford

University of Cambridge University of London..

University of Durham Universty of Liverpool

Victoria University of Manchester

University of Ieeds

University of Sheffield England

Society of Apothecaries of London University of Birmingham

Conjoint Examining Board in

\section{QUALIFICATION}

\section{M.B., B.Ch. (This degree} is only obtainable after graduating in Arts.)

M.B., B.C.

M.B.

M.B.

M.B., B.Ch.

M.B., Ch.B.

M.B., Ch.B

M.B., Ch.B.

M.B., Ch.B.

M.R.C.S., L.R.C.P.

\section{SCOTLAND.}

University of Edinburgh ‥ M.B., Ch.B

University of Glasgow … M.B., Ch.B.

University of Aberdeen ... M.B., Ch.B.

University of St. Andrews ... M.B., Ch.B.

Conjoint Examining Board in L.R.C.S.Edin. L.R.C.P. Scotland

Edin., \& L.F.P.S.Glasg. IRELAND.

University of Dublin ..

$$
\begin{aligned}
& \text { M.B., B.Ch., B.A.O. (These } \\
& \text { degrees are only con- } \\
& \text { ferred on graduates in } \\
& \text { Arts.) }
\end{aligned}
$$

Royal University of Ireland ... M.B., B.Ch.

Conjoint Examining Board in Ireland

\section{L.R.C.P.I., L.R.C.S.I.}

Apothecaries' Hall of Ireland L.A.H.Dub.

Many of these examining bodies lay down certain restrictions as to residence, and it is therefore important that an intending candidate should learn what these restrictions are by applying to the examining body from which he wishes to obtain his qualification.

\section{THE CONJOINT COURSE.}

THe student who is desirous of taking the Conjoint qualification-that is to say, the double diploma of Member of the Royal College of Surgeons and Licentiate of the Royal College of Physicians-has to comply with the regulations laid down for medical qualifications generally, a summary of which has already been given.

When registered as a medical student the candidate has to show that he has attended courses of lectures and practical work in chemistry and biology, before he is allowed to proceed to either the first or the second part of his " first." If he wishes to take the third part at the same time he has to show proof that he has been properly instructed in practical pharmacy by a recognised chemist or registered practitioner. The subjects for the first examination are chemistry and chemical physics, biology and pharmacy. In the first part the examination is partly written and partly practical; in the second and third parts it is wholly oral. The paper in chemistry does not usually present any difficult features; the questions set are straightforward and not tricky, and the examination tests fairly accurately the candidate's knowledge within the limits prescribed. These limits are not very wide, but they are sufficient for all practical purposes and the knowledge which the student acquires while he works for the first part of his first will be of real service to him later on. The practical part of the examination consists of simple quantitative and qualitative analysis, and candidates usually find the test a comparatively easy one. Assuming that the student has had some preliminary training, such as can usually be obtained at the general schools from which he passes his preliminary, he ought not to take a longer time than six months to pass the first and second parts of the first. Many students take both parts after only three months' work.

The biology required for the first examination is exceedingly elementary. A knowledge of the structure (acquired by actual dissection) of the frog, the 